\title{
Solving Short-Term Cascaded Hydrothermal Scheduling Problem Using Modified Cuckoo Search Algorithm
}

\author{
Thang Trung Nguyen ${ }^{1 *}$ and Dieu Ngoc $\mathrm{Vo}^{2}$ \\ ${ }^{1}$ Power System Optimization Research Group, Faculty of Electrical and \\ Electronics Engineering, Ton Duc Thang University, Vietnam \\ 19, Nguyen Huu Tho str., $7^{\text {th }}$ dist., HCM City, Vietnam \\ ${ }^{2}$ Department of Power Systems, HCM City University of Technology, VNU-HCM \\ 268 Ly Thuong Kiet str., 10th dist., HCM City, Viet Nam \\ nguyentrungthang@tdt.edu.vn,vndieu@gmail.com
}

\begin{abstract}
This paper presents a modified cuckoo search algorithm (MCSA) for solving shortterm cascaded hydrothermal scheduling (ST-CHTS) problem. The short-term cascaded hydrothermal scheduling is to determine the optimal operation for thermal plants and a cascaded reservoir system while satisfying all constraints including electrical constraints of both hydro and thermal plants and hydraulic constraints of reservoirs. The MCSA has been developed by modifying the search strategy via Lévy flights to improve the performance of the conventional cuckoo search algorithm. The proposed method has been widely and successfully applied to many optimization problems in engineering fields; however, this is first time employed to search for the optimal solution of the ST-CHTS problem. The proposed method has been tested on two systems where thermal plants with nonconvex fuel cost function and a cascaded reservoir system are taken into account. The result comparison from the MCSA compared to other methods reported in the literature has revealed that the proposed MCSA is very efficient for solving the ST-CHTS problem.
\end{abstract}

Keywords: Modified Cuckoo search algorithm; cascaded hydropower plants; Lévy flights

\section{Introduction}

A power system mainly consists of thermal plants and hydropower plants to supply electricity to load demand. Therefore, optimal operation of a hydrothermal system is very important once the electricity generation cost is minimized. One of the problems regarding to hydrothermal scheduling problem is short-term cascaded hydrothermal scheduling problem where the main task is to determine the power output of each available power plant so that the generation fuel cost is minimized over an optimization interval such as a single day and a week while electrical and hydraulic constraints including power balance, limits on generations, water discharge rate, and as well as reservoir storage limits are exactly met [1].

In recent decades, several artificial intelligence algorithms, such as genetic algorithm (GA) [2-3], two-phase neural network [4], evolutionary programming technique (EP) [5], particle swarm optimization (PSO) [6-7], differential evolution [8-9], and clonal selection algorithm (CSA) [10] have been widely and successfully applied for solving the STCHTS problems where quadratic and/or nonconvex fuel cost function of thermal units are considered. Among the methods, GA is the worst one since it obtains very high fuel cost, high constraint violation and long execution time. Hydro turbine with prohibited zone and

* Corresponding Author 
nonconvex fuel cost function of thermal units are considered in the study [5]; however, the article only tested the performance of several modified versions of EP. The reported results have shown that several disadvantages of the EP such as poor solution quality and long computational time are not tackled yet.

Cuckoo search algorithm (CSA), a very efficient meta-heuristic algorithm, was developed by Yang and Deb in 2009 [11]. The CSA has been successfully and widely applied to several engineering fields, especially electrical engineering such as economic load dispatch [12], short-term fixed head hydrothermal scheduling [13], short-term cascaded hydrothermal scheduling [14]. The CSA was regarded as a few control parameter method with high successful rate and good solution quality since it has obtained better results in terms of fuel cost and execution time than other meta-heuristic such as GA, improved GA, PSO, DE as well as several recent new methods like artificial immune system and hybrid algorithms. Despite of the strong points, the CSA has just been pointed out by Walton et al. [15] that it also possesses several drawbacks including local solution search based on random walk and long execution time. Finally, the authors suggested a modified version of the CSA, called modified CSA (MCSA). The MCSA was built by improving the search ability of the CSA with several modifications. Namely, in the first new solution generation via Lévy flights all initial eggs are ranked and classified into two groups where top group consists of better eggs and abandoned group contains other eggs, and the updated step size associated with the Lévy flights is adaptive for the two groups at each iteration. The MCSA fast attracted intentions from researchers to deal with optimization problems such as Benchmark optimization functions [15], design process of integrated power systems for modern and energy self-sufficient farms [16] and short-term fixed hydrothermal scheduling [17].

In this paper, the modified Cuckoo Search Algorithm (MCSA) is first proposed for solving short-term cascaded hydrothermal scheduling problem considering cascaded hydropower plants. The effectiveness of the proposed MCSA methods has been tested on one system and the obtained results have been compared to those from methods reported in the paper.

\section{Problem Formulation}

In this section, the mathematical formulation of the short-term HTS problem consisting of $N_{l}$ thermal units and $N_{2}$ hydro units scheduled in $M$ time sub-intervals with $t_{m}$ hours for each is formulated. The objective of the problem is to minimize total cost of thermal units subject to the system and unit constraints.

The mathematical model of the problem is formulated as follows:

$F=\sum_{m=1}^{M} \sum_{i=1}^{N_{1}} t_{m}\left(a_{s i}+b_{s i} P_{s i, m}+c_{s i} P_{s i, m}^{2}\right)$

where $a_{s i}, b_{s i}, c_{s i}$ are fuel cost coefficients of thermal plant $i$; $P_{s i, m}$ is the power output of thermal plant i at subinterval $m$.

subject to:

\subsection{Active Power Balance Constraint}

$\sum_{i=1}^{N_{1}} P_{s i, m}+\sum_{j=1}^{N_{2}} P_{h j, m}-P_{L, m}-P_{D, m}=0$

where $P_{L, m}$ and $P_{D, m}$ are load demand and transmission loss at subinterval $m ; \mathrm{P}_{\mathrm{hj}, \mathrm{m}}$ is the power output of hydro plant $\mathrm{j}$ at subinterval $m$ and is determined by

$P_{h j, m}=C_{1 h j}\left(V_{j, m}\right)^{2}+C_{2 h j}\left(Q_{j, m}\right)^{2}+C_{3 h j} Q_{j, m} V_{j, m}+C_{4 h j} V_{j, m}+C_{5 h j} Q_{j, m}+C_{6 h j}$ 
where $\mathrm{C}_{1 \mathrm{hj}}, \mathrm{C}_{2 \mathrm{hj}}, \mathrm{C}_{3 \mathrm{hj}}, \mathrm{C}_{4 \mathrm{hj}}, \mathrm{C}_{5 \mathrm{hj}}, \mathrm{C}_{6 \mathrm{hj}}$ are the coefficients of the jth hydropower plant

\subsection{Hydraulic Continuity Constraint}

$V_{j, m-1}-V_{j, m}+I_{j, m}-Q_{j, m}-S_{j, m}+\sum_{i=1}^{N u} \sum_{m=1}^{M}\left(Q_{i, m-\tau_{i, j}}+S_{i, m-\tau_{i, j}}\right)=0$

where $V_{j, m}, I_{j, m}$ and $S_{j, m}$ are reservoir volume, water inflow and spillage discharge rate of $j$ th hydropower plant in $m t h$ interval. $\tau_{i, j}$ is the water delay time between reservoir $j$ and its up-stream $i$ at interval $m$ and $\mathrm{Nu}$ is the set of up-stream unitsdirectly above hydro-plant $j$.

\subsection{Initial and Final Volume Constraints}

$V_{j, 0}=V_{j, \text { initial }} ; V_{j, M}=V_{j, E n d}$

\subsection{Upper and Lower Volume Constraints}

$V_{j, \min } \leq V_{j, m} \leq V_{j, \text { max }} ; j=1,2, \ldots, N_{2} ; m=1,2, \ldots, M$

where $V_{j, \max }$ and $V_{j, \min }$ are the maximum and minimum reservoir storage of the hydro plant $j$, respectively.

\subsection{Water Discharge Rate Limits}

$Q_{j, \text { min }} \leq Q_{j, m} \leq Q_{j, \text { max }} ; j=1,2, \ldots, N_{2} ; m=1,2, \ldots, M$

where $Q_{j, \max }$ and $Q_{j, \min }$ are the maximum and minimum water discharge of the hydro plant $j$.

\subsection{Upper and Lower Generation Constraints}

$P_{s i, \mathrm{~min}} \leq P_{s i, m} \leq P_{s i, \mathrm{max}} ; i=1,2, \ldots, N_{1} ; m=1,2, \ldots, M$

$P_{h j, \text { m in }} \leq P_{h j, m} \leq P_{h j, \text { max }} ; j=1,2, \ldots, N_{2} ; m=1,2, \ldots, M$

where $P_{s i, \max }, P_{s i, \min }$ and $P_{h j, \max }, P_{h j, \min }$ are maximum, minimum power output of thermal plant $i$ and hydro plant $j$, respectively.

\section{Modified Cuckoo Search Algorithm for ST-CHTS Problems}

\subsection{Modified Cuckoo Search Algorithm}

The conventional CSA is comprised of three main parts including initialization, the first new solution generation via Lévy flights and the second new solution generation via the action of alien egg discovery. The initialization part is inspired from the laying and dumping egg of cuckoo birds in other species nests while the two new solution generations are inspired from final result of the cuckoo eggs after dumped in the host nest. As the host bird does not identify the alien egg, the egg will be hatched and carried over to the next generation. This phenomenon results in the first generation. Besides, there will be a fraction of the fixed number of eggs discovered as alien eggs and abandoned by the host bird. Obviously, a difference between the first and the second generations is that all 
the eggs from the initialization are newly generated in the first time via the Lévy flights while only a fraction of eggs is newly generated in the second time.

The new solutions generated via Lévy flights are obtained as below [14]:

$X_{i}^{\text {new }}=X_{i}+\alpha \cdot\left(X_{\text {best }}-X_{i}\right)\left(v \times \frac{\sigma_{x}(\beta)}{\sigma_{y}(\beta)}\right)$

where $X_{\text {best }}$ and $X_{i}$ are the best egg and the ith egg among the number of eggs; $\alpha>0$ is an updated step size.

The value of $\alpha$ has a significant influence on the final solution because it will lead to different new solutions as it is set to different values. If this parameter is set to a high value, there is a huge difference between the old and new solutions and the optimal solution is either obtained fast or omitted. As the current iteration is high, the new obtained solution should be searched nearby the previous solution. However, the method has to find the optimal solution in a large search zone for this set value which may not reach the best optimal solution. Based on the analyzed drawback of the conventional CSA, it is clearly better to search for the optimal solution in a small zone as the iteration counter is increased to the maximum number of iterations which is predetermined for the iterative process [15].

The MCSA [15] has been focusing on the updated step size and improving the quality of all solutions generated via the Lévy flights. In the MCSA, all the eggs are ranked and classified into the top group with better quality eggs and the abandoned group with worse quality eggs before applying the first new solution generation via the Lévy flights. The abandoned group only focuses on the step size $\alpha$ which is decreased as the current iteration is increased. It is more complicated as generating new solution for the top group since it needs information exchanging between two eggs, one is randomly picked and the other is picked in order. Three cases may happen to the two picked eggs as follows: 1) the same egg is picked twice; 2) the two eggs have the same fitness function value; and 3) the randomly picked egg has lower or higher fitness value than the predetermined egg.

\subsection{Units Calculation of Power Output for Slack Thermal Unit}

In the MCSA most variables are first determined excluding slack ones, which are used to exactly meet power balance constraint (2) and hydraulic continuity constraint (4). The slack variables consisting of the water discharge of $j$ th reservoir at subinterval $\mathrm{M}, Q_{j, M, d}$ and power output of thermal unit 1 at subinterval $\mathrm{m}, P_{s l, m}$ are obtained as follows [14]:

$P_{s 1, m}=P_{D, m}+P_{L, m}-\sum_{i=2}^{N_{1}} P_{s i, m}-\sum_{j=1}^{N_{2}} P_{h j, m}$
$Q_{j, M, d}=V_{j, 0}-V_{j, M}+\sum_{m=1}^{M} I_{j, m}-\sum_{m=1}^{M-1} Q_{j, m}-\sum_{m=1}^{M} S_{j, m}+\sum_{i=1}^{N u} \sum_{m=1}^{M}\left(Q_{i, m-\tau_{i, j}}+S_{i, m-\tau_{i, j}}\right)=0$

\subsection{Implementation of the Modified Cuckoo Search Algorithm}

3.3. 1 Initialization: Similar to other meta-heuristic algorithms, each nest of Np nests is represented by $X_{d}=\left[P_{s i, m, d} Q_{j, m, d}\right]\left(d=1, \ldots, N_{p}\right)$. Each nest $X_{d}$ is randomly initialized where $P_{s i, \min } \leq P_{s i, m, d} \leq P_{s i, \max }\left(i=2, \ldots, N_{l} ; m=1, \ldots, M\right)$ and $Q_{j, \min } \leq Q_{j, m, d} \leq Q_{j, \max }\left(j=1, \ldots ., N_{2}\right.$; $m=1, \ldots ., M-1)$.

Using (4), the reservoir volume at $m$ th subinterval is obtained by

$V_{j, m}=V_{j, m-1}+I_{j, m}-Q_{j, m}-S_{j, m}+\sum_{i=1}^{N_{u}}\left(Q_{j, m-\tau_{i, j}}+S_{i, m-\tau_{i, j}}\right)$

The $Q_{j, M, d}$ is obtained by (12) and hydro generations can be then calculated using (3). The slack thermal unit is obtained using (11). 
Based on the initial population of nests, the fitness function to be minimized corresponding to each nest for the considered problem is calculated.

$$
F T_{d}=\left\{\begin{array}{l}
\sum_{m=1}^{M} \sum_{i=1}^{N 1} F_{i}\left(P_{s i, m, d}\right)+K_{s} \sum_{m=1}^{M}\left(P_{s 1, m, d}-P_{s 1}^{\lim }\right)^{2}+K_{V} \sum_{j=1}^{N 2} \sum_{m=1}^{M-1}\left(V_{j, m, d}-V_{j}^{\lim }\right)^{2} \\
+K_{Q} \sum_{j=1}^{N 2}\left(\left(Q_{j, M, d}-Q_{j}^{\lim }\right)^{2}\right)+K_{h} \sum_{j=1}^{N 2} \sum_{m=1}^{M}\left(P_{h j, m, d}-P_{h j}^{\lim }\right)^{2}
\end{array}\right\}
$$

where $K_{s}$ and $K_{h}$ are respectively penalty factors for the slack thermal unit 1 and all hydro units; $K_{V}$ and $K_{Q}$ are respectively penalty factors for reservoir volume over $M-1$ subintervals and water discharge at the subinterval $\mathrm{M}$;

The limits of variables in (14) are obtained as below.

$$
\begin{aligned}
& P_{s 1}^{\lim }= \begin{cases}P_{s 1, \mathrm{max}} & \text { if } P_{s 1, m, d}>P_{s 1, \mathrm{max}} \\
P_{s 1, \mathrm{~min}} & \text { if } P_{s 1, m, d}<P_{s 1, \mathrm{~min}} ; m=1, \ldots, M \\
P_{s 1, m, d} & \text { otherwise }\end{cases} \\
& V_{j}^{\lim }=\left\{\begin{array}{lll}
V_{j, \mathrm{max}} & \text { if } V_{j, m, d}>V_{j, \mathrm{max}} \\
V_{j, \mathrm{~min}} & \text { if } V_{j, m, d}<V_{j, \mathrm{~min}} & ; j=1, \ldots, N_{2} ; \\
V_{j, m, d} & \text { otherw ise } \quad m=1, \ldots, M-1
\end{array}\right. \\
& Q_{j}^{\lim }= \begin{cases}Q_{j, \max } & \text { if } Q_{j, M, d}>Q_{j, \max } \\
Q_{j, \text { min }} & \text { if } Q_{j, M, d}<Q_{j, \text { min }} ; j=1, \ldots, N_{2} \\
Q_{j, M, d} & \text { otherwise }\end{cases} \\
& P_{h j}^{\lim }= \begin{cases}P_{h j, \mathrm{max}} & \text { if } P_{h j, m, d}>P_{h j, \mathrm{max}} \\
P_{h j, \mathrm{~min}} & \text { if } P_{h j, m, d}<P_{h j, \mathrm{~min}} \quad ; j=1, \ldots, N_{2} ; \\
P_{h j, m, d} & \text { otherwise } \quad m=1, \ldots, M\end{cases}
\end{aligned}
$$

As described above, in the MCSA all nests are first sorted in the descending order of their fitness function value and then classified into two groups. The nests with high fitness function value Xabandoned $_{d}$ are put in the abandoned group and the other ones Xtop $_{d}$ are put in the top group. A nest which is randomly picked among the $X t_{o p} p_{d}$ nests is called $X_{t o p}$ and another one with the best quality is named Xbest $t_{d}$. The two new solution generations are respectively described as below.

\subsubsection{Generation of New Solution via Lévy Distribution, Cauchy Distribution and} Gaussian Distribution: The first new solution generation via the Lévy flights

a) New solution generation for the abandoned group

Based on the modification applied to the abandoned eggs $\left(d=N o t o p+1, \ldots, N_{p}\right.$ where Notop and $N p$ are the number of eggs in the top group and in the initial population, respectively), the optimal path for the Lévy flights is calculated using Mantegna's algorithm as follows

Xabandoned ${ }_{d}^{\text {new }}=$ Xabandoned $_{d}+\alpha \times$ rand $_{1}+\Delta X$

where rand $_{1}$ is the distributed random number in $[0,1]$, the step size $\alpha$ is determined by $\frac{1}{\sqrt{G}}$ where $G$ is the current iteration number, and $\Delta X$ is obtained by:

$\Delta X=v \times \frac{\sigma_{x}(\beta)}{\sigma_{y}(\beta)} \times\left(\right.$ Xabandoned $_{d}-$ Xbest $) ;$

b) New solution generation for the top egg group 
The modification is applied to the eggs in the top group $(d=1, \ldots, N o t o p)$. The optimal path for the Lévy flights is calculated using Mantegna's algorithm as follows:

$X$ top $_{d}^{\text {new }}=X$ top $_{d}+\alpha \times$ rand $_{2} \times \Delta X$

where rand $_{2}$ is the distributed random numbers in $[0,1]$.

The value of $\alpha$ and $\Delta X$ will be determined depending on the considered cases as follows:

- Case 1: The same egg is picked twice

$\Delta X$ top $p_{d}^{\text {new }}=v \times \frac{\sigma_{x}(\beta)}{\sigma_{y}(\beta)} \times\left(X t o p_{d}-X\right.$ best $)$

where $\alpha=1 / G^{2}$.

- Case 2: Both eggs have the same fitness value function

$\Delta X$ top ${ }_{d}^{\text {new }}=\left(X t o p_{d}-X\right.$ top $\left.p_{r}\right) / 2$

where $\alpha=1$.

- Case 3: The random egg has lower fitness than egg $d$

$\Delta X_{d}^{n e w}=\left(X t o p_{r}-X t o p_{d}\right) / \varphi$

or the random egg has higher fitness than egg $d$

$\Delta X=\left(X t o p_{d}-X t o p_{r}\right) / \varphi$

where $\alpha=1$ and $\varphi=(1+\sqrt{5}) / 2$.

3.3.1.1 The Second New Solution Generation via Discovery of Alien Eggs: Similar to the conventional CSA, the second new solution generation via discovery of alien egg is also employed in the MCSA but all eggs of the top group and abandoned group are combined into one group first. The new solution due to this action can be found as follows:

$X_{d}^{d i s}=$ Xbest $_{d}+K \times \Delta X_{d}^{d i s}$

where $K$ is the updated coefficient determined based on the probability of a host bird to discover an alien egg in its nest [11] and $\Delta X_{d}^{\text {dis }}$ is the increased value [11].

The new solutions can violate their limits and need to be redefined by:

$\begin{aligned} & P_{s i, m, d}= \begin{cases}P_{s i, \mathrm{max}} & \text { if } P_{s i, m, d}>P_{s i, \mathrm{max}} ; i=2, \ldots, N_{1} \\ P_{s i, \mathrm{~m} \text { in }} & \text { if } \quad P_{s i, m, d}<P_{s i, \mathrm{~min}} \quad m=1, \ldots, M \\ P_{s i, m, d} & \text { otherwise }\end{cases} \\ & Q_{j, m, d}=\left\{\begin{array}{lll}Q_{j, \mathrm{max}} & \text { if } Q_{j, m, d}>Q_{j, \mathrm{max}} ; j=1, \ldots, N_{2} \\ Q_{j, \mathrm{~min}} & \text { if } Q_{j, m, d}<Q_{j, \mathrm{~min}} \quad m=1, \ldots, M-1 \\ Q_{j, m, d} & \text { otherwise }\end{array}\right.\end{aligned}$

The power output of $N_{2}$ hydro units and the slack thermal unit are then obtained, respectively. The fitness value is respectively calculated using equations (14). The nest corresponding to the best fitness function is then set to the best nest Xbest.

3.3.2 Stopping Criteria: The above algorithm is stopped when the maximum number of iterations is reached.

\section{Overall Procedure}

The overall procedure of the proposed MCSA for solving the ST- CHTS problem is described as follows. 
Step 1: Select parameters including number of nests $N_{p}$, probability of alien eggs to to be abandoned $P_{a}$, the ratio of the number of eggs in top group to that in abandoned group and maximum number of iterations $N_{\max }$.

Step 2: Initialize a population of $N_{p}$ host nests as in Section 4.1 and calculate the slack unit 1 using eq. (11). Set the initial iteration counter $G=1$.

Step 3: Evaluate the fitness function using (14) to evaluate and classify the eggs into two groups including abandoned group, Xabandoned $_{d}(\mathrm{~d}=$ Notop $+1, \ldots, N p)$ and top group, $X_{\text {Top }}(\mathrm{d}=1, \ldots$, Notop $)$. The egg with the lowest fitness function value is set to Gbest in the population.

Step 4: Generate new solutions via Lévy flights for abandoned group as described in Section 4.2.1 and calculate the slack unit 1 using eq. (11).

Step 5: Generate new solutions via Lévy flights for top group as described in Section 4.2.2 and calculate the slack unit 1 using eq. (11).

Step 6: Put the new eggs in top group and abandoned group in the integrated group and evaluate all the eggs to determine the best egg with the lowest fitness function.

Step 7: Generate new solutions via the discovery of alien eggs as described in Section 4.3 and calculate the slack unit 1 using eq. (11).

Step 8: Evaluate the fitness function using (14) to determine the best egg, Gbest.

Step 9: If $\mathrm{G}<\mathrm{Gmax}, \mathrm{G}=\mathrm{G}+1$ and go back to step 3. Otherwise, terminate the iterative procedure.

\section{Numerical Results}

The proposed MCSA has been implemented for solving four hydrothermal systems classified into two cases, where case 1 considers two systems with four cascaded hydropower plants and one thermal plant with quadratic fuel cost function, and case 2 considers two systems with four cascaded hydropower plants and three thermal plants with nonconvex fuel cost function. All hydrothermal systems are scheduled in 24 onehour subintervals. The proposed MCSA is coded in Matlab platform and run fifty independent trials on a $1.8 \mathrm{GHz}$ PC with $4 \mathrm{~GB}$ of RAM.

Table 1. Results Obtained by MCSA

\begin{tabular}{|c|c|c|c|c|c|}
\hline$P_{a}$ & $\begin{array}{c}\text { Min. total } \\
\text { cost }(\$)\end{array}$ & $\begin{array}{c}\text { Aver. total } \\
\text { cost }(\$)\end{array}$ & $\begin{array}{c}\text { Max. total } \\
\text { cost }(\$)\end{array}$ & $\begin{array}{c}\text { Std. } \\
\text { dev. } \\
(\$)\end{array}$ & $\begin{array}{c}\text { Avg. } \\
\text { CPU } \\
(\mathrm{s})\end{array}$ \\
\hline 0.1 & 923538.7 & 923837.8 & 924115.4 & 136.2 & 230 \\
\hline 0.2 & 923029.5 & 923465.4 & 923725.2 & 142.3 & 228 \\
\hline 0.3 & 922927.5 & 923366.4 & 923655.5 & 166.0 & 243 \\
\hline 0.4 & 923002.7 & 923290.8 & 923547.5 & 141.4 & 236 \\
\hline 0.5 & 922831.4 & 923134.1 & 923615.4 & 175.0 & 233 \\
\hline 0.6 & 922773.6 & 923141.9 & 923626.2 & 196.2 & 234 \\
\hline 0.7 & 922788.4 & 923138.1 & 923560.8 & 171.8 & 237 \\
\hline 0.8 & 922786.9 & 923153.8 & 923634.4 & 199.4 & 235 \\
\hline 0.9 & 922839.5 & 923390.9 & 923946.4 & 252.5 & 232 \\
\hline
\end{tabular}

\subsection{Control Parameter Selection}

The proposed MCSA consists of three conventional CSA parameters including the number of nests $N_{p}$, maximum number of iterations $N_{\max }$ and the probability of an alien egg to be discovered $P_{a}$ and one individual parameter of the MCSA, the ratio of the number of eggs in top group to that in abandoned group. Among the parameters, the number of nests and the maximum number of iterations directly impact on the obtained 
results in terms of total generation cost and computational time. As the parameters are set to high values the better cost is obtained but the MCSA spends longer computational time terminating the search process, and vice versa. On the contrary, the probability of the eggs to be discovered does not influence the computational time but obtained generation cost. The optimal value of the parameter will be determined after setting to a range from 0.1 to 0.9 with a step of 0.1 and evaluating the minimum total generation cost obtained. Normally, the optimal value of the parameter falls in the two zones, $[0.1,0.5]$ and $[0.5$, 0.9 ] but the particular value is not fixed at one value. Besides, the ratio of the top nests to the abandoned nests also affect the solution quality. It has been suggested 1:3 in the studies $[15,17]$ and good results have been obtained. Therefore, the value is retained in the paper.

For implementation of the proposed MCSA, the number of nests and the maximum number of iterations are set to 100 and 15000 meanwhile the ratio of top nests to abandoned nests is $25: 75$ and the probability is set in range of 0.1 to 0.9 with a step of 0.1 .

\subsection{Obtained Results and Discussions}

Case 1: Two systems with quadratic fuel cost function of thermal units.

This case considers two four-hydropower plant and one-thermal plant systems, where the data of system 1 and system 2 are respectively taken from [2] and [4]. The two systems have the same data of hydropower plants excluding transportation delay times and different fuel cost function of thermal unit.

The obtained results in terms of minimum total cost, maximum total cost, average total cost, standard deviation cost and average computational time for system 1 are given in Table 1. Clearly, the lowest value of minimum cost, average cost, maximum cost and standard deviation cost are obtained at $\mathrm{Pa}=0.6,0.5,0.4$ and 0.1 , respectively. As carefully observed from the minimum total cost column, the minimum total costs in range [0.6, 0.9] are less than those in range $[0.1,0.5]$ as mentioned in section selection of parameters.

Table 2. Obtained Result Comparison for System 1 of Case 1

\begin{tabular}{|l|c|c|c|c|}
\hline \multicolumn{1}{|c|}{ Method } & $\begin{array}{c}\text { Min cost } \\
(\$)\end{array}$ & $\begin{array}{c}\text { Average } \\
\text { cost }(\$)\end{array}$ & $\begin{array}{c}\text { Max cost } \\
(\$)\end{array}$ & $\begin{array}{c}\text { Avg. } \\
\text { time (s) }\end{array}$ \\
\hline GA[2] & 942600 & 946609.1 & 951087 & 1920 \\
\hline BCGA [3] & $926,922.71$ & - & - & - \\
\hline RCGA [3] & $925,940.03$ & - & - & - \\
\hline CEP[5] & 930166.25 & 930373.23 & 930927.01 & 2292.1 \\
\hline FEP[5] & 930267.92 & 930897.44 & 931396.82 & 1911.2 \\
\hline IFEP[5] & 930129.82 & 930290.13 & 930881.92 & 1033.2 \\
\hline GCPSO [6] & 927288.4 & 936717.1 & 972658.3 & 182.4 \\
\hline GWPSO [6] & 930622.5 & 940036.3 & 951253.2 & 129.1 \\
\hline LCPSO [6] & 925618.5 & 926651.4 & 928219.8 & 103.5 \\
\hline LWPSO [6] & 925383.8 & 926352.8 & 927240.1 & 82.9 \\
\hline EPSO [7] & $922,904.00$ & - & - & - \\
\hline DE [9] & $923,991.08$ & - & - & - \\
\hline MCSA & 922773.6 & 923141.9 & 923626.2 & 234 \\
\hline
\end{tabular}




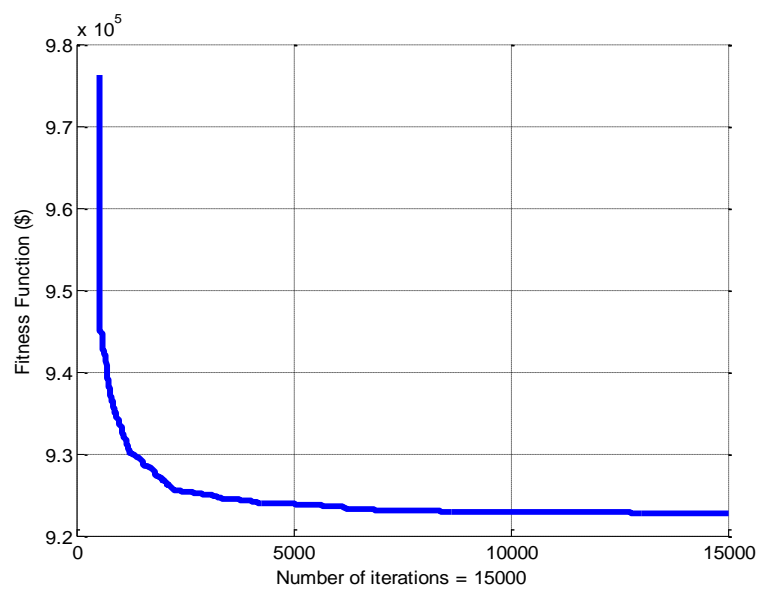

Figure 1. Convergence Characteristic for System 1 of Case 1

Table 3. Obtained Result Comparison for System 2 of Case 1

\begin{tabular}{|l|c|c|}
\hline \multicolumn{1}{|c|}{ Method } & $\begin{array}{c}\text { Min cost } \\
(\$)\end{array}$ & $\begin{array}{l}\text { Avg. time } \\
(\mathrm{s})\end{array}$ \\
\hline TPNN [9] & 154808.5 & - \\
\hline ALM[9] & 154739 & - \\
\hline PSO [18] & 154705 & - \\
\hline ISAPSO[18] & 154594.7 & - \\
\hline MCSA & 154594.4 & 231 \\
\hline
\end{tabular}

Table 4. Optimal Solution Obtained for System 1 of Case 1

\begin{tabular}{|c|c|c|c|c|c|}
\hline \multirow{2}{*}{ Hour } & \multicolumn{3}{|c|}{ Water discharge $\left(\times 10^{4} \mathrm{~m}^{3}\right)$} & \multirow{2}{*}{$\mathrm{P}_{\mathrm{S}}(\mathrm{MW})$} \\
\cline { 2 - 5 } & plant 1 & plant 2 & plant 3 & plant 4 & \\
\hline 1 & 9.6262 & 6.5951 & 29.9542 & 13 & 1031.6022 \\
\hline 2 & 9.6969 & 6.2608 & 29.9972 & 13.0007 & 1065.1271 \\
\hline 3 & 8.9499 & 6.0978 & 28.2986 & 13.0029 & 1052.8443 \\
\hline 4 & 8.4563 & 6.2135 & 29.9574 & 13 & 1000.7727 \\
\hline 5 & 8.4734 & 6.0829 & 18.1989 & 13.0009 & 954.3588 \\
\hline 6 & 8.1653 & 6.1552 & 17.5079 & 13 & 1052.2903 \\
\hline 7 & 8.0089 & 6.4848 & 16.9502 & 13 & 1272.1805 \\
\hline 8 & 8.607 & 7.4101 & 15.5737 & 13.0058 & 1591.4313 \\
\hline 9 & 8.3575 & 7.5467 & 15.8458 & 13.0272 & 1827.1237 \\
\hline 10 & 8.7161 & 7.5915 & 15.4544 & 13 & 1898.2346 \\
\hline 11 & 8.6895 & 8.1715 & 15.2537 & 13.0571 & 1798.2119 \\
\hline 12 & 8.5758 & 8.4514 & 15.5336 & 13 & 1874.8588 \\
\hline 13 & 8.6059 & 8.6845 & 14.4437 & 13.3912 & 1782.4709 \\
\hline 14 & 8.4309 & 8.6645 & 16.0137 & 15.5024 & 1734.5570 \\
\hline 15 & 8.2826 & 8.7114 & 17.0762 & 14.8096 & 1671.9811 \\
\hline 16 & 8.0756 & 9.0671 & 16.3803 & 15.5968 & 1601.5177 \\
\hline 17 & 7.7806 & 9.1441 & 17.2503 & 15.2543 & 1669.6097 \\
\hline 18 & 7.7112 & 9.4308 & 14.9736 & 15.6018 & 1670.1957 \\
\hline 19 & 7.7541 & 10.4742 & 17.1273 & 16.6918 & 1762.5296 \\
\hline 20 & 7.5693 & 10.9308 & 12.3502 & 16.8751 & 1793.9829 \\
\hline 21 & 7.609 & 11.3778 & 10.0756 & 18.4505 & 1742.4466 \\
\hline 22 & 7.3763 & 10.2376 & 10.203 & 19.6986 & 1624.0885 \\
\hline 23 & 6.0615 & 10.6778 & 10.5695 & 20.5374 & 1360.5140 \\
\hline 24 & 5.4202 & 11.5381 & 10.3758 & 22.4368 & 1106.7223 \\
\hline & & & & & \\
\hline
\end{tabular}


Table 2 reports the comparison of result for system 1 obtained by the proposed MCSA and other methods including Genetic algorithm in [2], Binary coded GA (BCGA) and real coded GA (RCGA) in [3], classical evolutionary programming (CEP), Fast EP (FEP) and improved Fast EP (IFEP) in [5], global constriction PSO (GCPSO), global weight factor PSO (GWPSO), Local constriction PSO (LCPSO) and local weight factor PSO (LWPSO) in [6], enhanced PSO (EPSO) in [7], and differential evolutionary (DE) in [9]. As observed from the table, the proposed method obtains better minimum cost, average cost and maximum cost than all methods available in the table. With respect to the computational time, the proposed method is faster than most methods excluding several versions of PSO in [6].

For system 2, The MCSA obtains the best minimum cost at $\mathrm{Pa}=0.4$ and the comparison of the best cost among the MCSA and other methods such as two-phase neural network (TPNN) and augmented Lagrange method (ALM) in [4], PSO and improved self adaptive PSO (ISAPSO) in [18] indicated in Table 3. Obviously, the MCSA gets much better solution than all methods excluding ISAPSO. There is no simulation time reported for these methods in the table.

Consequently, the proposed is very efficient for determining the optimal solution for the ST-CHTS problem with quadratic fuel cost function of thermal units. The optimal solution obtained and cost convergence characteristic by the proposed method for system 1 of case 1 are given in Table 4 and Figure 1.

Case 2: Two systems with nonconvex fuel cost function of thermal units.

Two test systems with four cascaded hydropower plants and three thermal plants with nonconvex fuel cost function are considered in this case. The data for the two systems are shown in [19] and [20], respectively. The best minimum cost that the MCSA obtain for system 1 and 2 are respectively at $\mathrm{Pa}=0.4$ and 0.7 . Table 5 presents the cost and simulation time obtained from the MCSA and evolutionary programming based interactive fuzzy satisfying method (EP-IFS) in [19], Simulated annealing (SA), evolutionary programming (EP) and Particle swarm optimization (PSO) in [21], differential evolution (DE) and Real coded genetic algorithm (RCGA) [20]. As observed from the table, the proposed MCSA obtains much less cost than all methods for both system 1 and system 2. Furthermore, the MCSA is also much faster than DE and RCGA. The DE and RCGA were coded on a Pentium IV, $3 \mathrm{GHz}$ computer. Other methods have not reported computational time and computer. The optimal solution obtained by the proposed MCSA for test system 1 of the case is given in Table 6 .

Table 5. Result Comparison for Case 2

\begin{tabular}{|l|c|c|c|c|}
\hline \multirow{2}{*}{ Method } & \multicolumn{2}{|c|}{ System 1 } & \multicolumn{2}{c|}{ System 2 } \\
\cline { 2 - 5 } & Cost (\$) & time (s) & Cost (\$) & time (s) \\
& & & & \\
\hline EP-IFS [19] & 45063 & - & - & - \\
\hline SA [21] & 47306 & - & - & - \\
\hline EP [21] & 45466 & - & - & - \\
\hline PSO [21] & 44740 & - & - & - \\
\hline DE [20] & - & - & 110810 & 2554.1 \\
\hline RCGA [20] & - & - & 112940 & 3156.5 \\
\hline MCSA & 43476 & 265 & 82790 & 254 \\
\hline
\end{tabular}


Table 6. Optimal Solution Obtained by MCSA for System 1 of Case 2

\begin{tabular}{|c|l|l|l|l|l|l|l|}
\hline Hour & $\begin{array}{c}\mathrm{Q}_{1} \\
\left(10^{4} \mathrm{~m}^{3}\right)\end{array}$ & \multicolumn{1}{|c|}{$\begin{array}{c}\mathrm{Q}_{2} \\
\left(10^{4} \mathrm{~m}^{3}\right)\end{array}$} & $\begin{array}{c}\mathrm{Q}_{3} \\
\left(10^{4} \mathrm{~m}^{3}\right)\end{array}$ & $\begin{array}{c}\mathrm{Q}_{4} \\
\left(10^{4} \mathrm{~m}^{3}\right)\end{array}$ & $\begin{array}{c}\mathrm{P}_{\mathrm{s} 1} \\
(\mathrm{MW})\end{array}$ & $\begin{array}{c}\mathrm{P}_{\mathrm{s} 2} \\
(\mathrm{MW})\end{array}$ & $\begin{array}{c}\mathrm{P}_{\mathrm{s} 3} \\
(\mathrm{MW})\end{array}$ \\
\hline 1 & 11.0785 & 9.0216 & 20.8281 & 8.6901 & 166.9237 & 112.1210 & 105.5187 \\
\hline 2 & 7.9614 & 11.4730 & 25.4217 & 12.9988 & 146.6096 & 207.7263 & 58.4384 \\
\hline 3 & 8.1518 & 6.0656 & 17.4365 & 8.6723 & 143.7393 & 158.7603 & 82.1866 \\
\hline 4 & 7.6695 & 6.4538 & 28.6583 & 6.4364 & 173.2124 & 118.4290 & 119.1982 \\
\hline 5 & 5.6920 & 10.4340 & 29.9667 & 7.1448 & 174.3974 & 97.0926 & 148.2308 \\
\hline 6 & 6.4772 & 6.2194 & 20.1650 & 6.4717 & 168.2794 & 253.6595 & 115.0933 \\
\hline 7 & 7.0610 & 6.2239 & 14.2006 & 17.4276 & 174.2758 & 205.5627 & 158.3464 \\
\hline 8 & 8.9154 & 7.0699 & 16.5039 & 10.0655 & 174.8861 & 288.8394 & 186.0087 \\
\hline 9 & 11.1597 & 8.8520 & 14.1511 & 15.3448 & 174.0448 & 258.7376 & 200.9088 \\
\hline 10 & 9.7363 & 7.4532 & 19.6945 & 16.2778 & 174.5498 & 265.2696 & 193.3469 \\
\hline 11 & 9.4101 & 6.9752 & 18.9932 & 17.5401 & 174.1841 & 268.3244 & 198.8700 \\
\hline 12 & 10.1721 & 8.1962 & 12.7667 & 19.7602 & 174.8303 & 298.6892 & 178.1246 \\
\hline 13 & 7.2956 & 8.7684 & 19.3153 & 19.3260 & 132.6811 & 269.5498 & 241.7761 \\
\hline 14 & 9.5292 & 7.8822 & 14.0598 & 12.8963 & 174.1711 & 265.2755 & 157.5033 \\
\hline 15 & 6.7198 & 9.0522 & 18.6116 & 18.5624 & 170.3292 & 236.8770 & 135.2646 \\
\hline 16 & 7.3888 & 11.8784 & 17.0181 & 18.8706 & 174.8517 & 247.3988 & 144.2679 \\
\hline 17 & 7.7947 & 8.0547 & 11.7512 & 13.1900 & 174.6419 & 240.2377 & 203.6291 \\
\hline 18 & 7.5930 & 6.8619 & 18.0923 & 18.7428 & 174.9501 & 291.2401 & 188.1574 \\
\hline 19 & 10.9461 & 12.0948 & 21.6544 & 18.5888 & 174.7640 & 230.1911 & 176.1318 \\
\hline 20 & 8.2280 & 9.2424 & 15.6426 & 18.7903 & 138.2875 & 274.4799 & 156.2832 \\
\hline 21 & 5.0168 & 11.3892 & 16.1661 & 18.3426 & 153.1518 & 142.1125 & 156.3306 \\
\hline 22 & 10.3895 & 7.8617 & 12.2974 & 19.9131 & 122.1920 & 200.5091 & 52.4855 \\
\hline 23 & 5.4065 & 7.5649 & 12.5606 & 19.3712 & 174.1068 & 122.1877 & 106.5570 \\
\hline 24 & 5.2070 & 6.9113 & 10.3930 & 18.3072 & 54.6683 & 182.6589 & 125.8985 \\
\hline & & & & & & & \\
\hline
\end{tabular}

\section{Conclusions}

In this paper, the proposed Modified Cuckoo Search Algorithm has been employed for determining the optimal solution for short-term hydrothermal system scheduling where cascaded hydropower plants and nonconvex objective are considered. The proposed MCSA has been tested on four systems and the results obtained are compared to those from several methods available in the paper. The comparison of generation fuel costs and computational time reveals that the MCSA is more effective and robust than these methods. Therefore, the proposed MCSA is one of the promising modern methods for solving the short-term cascaded hydrothermal scheduling problem where thermal units and a cascaded power plant system are connected.

\section{References}

[1] A. J. Wood, and B.F. Wollenberg, "Power Generation, Operation and Control", John Wiley \& Sons, New York, 1984.

[2] S.O. Orero and M.R. Irving, "A genetic algorithm modeling framework and solution technique for short termoptimal hydrothermal scheduling", IEEE Trans. Power Syst. (1998), vol. 13, pp. 501-518.

[3] S. Kumar, R. Naresh, "Efficient real coded genetic algorithm to solve the non-convex hydrothermal scheduling problem,” Int. J. Electr. Power Energ. Syst. (2007), vol. 29, pp. 738-747.

[4] R. Naresh and J. Sharma, "Two-phase neural network based solution technique for short term hydrothermal scheduling,” IEE Proc-Gener. Transm. Distrib. (1999), Vol. 146, No. 6, pp. 657 - 663.

[5] N. Sinha, R. Chakrabarti, and P. K. Chattopadhyay, "Fast evolutionary programming techniques for short-term hydrothermal scheduling," IEEE Transactions on Power Systems (2003), vol.18, pp.214-220. 
[6] B. Yu, X. Yuan, J. Wang, "Short-term hydro-thermal scheduling using particle swarm optimization method," Energy Conversion and Management (2007), vol. 48, pp. 1902-1908.

[7] X. Yuan, L. Wang, Y. Yuan, "Application of enhanced PSO approach to optimal scheduling of hydro system,” Energ. Convers. Manag. (2008), vol. 49, pp. 2966-2972.

[8] L. Lakshminarasimman, S. Subramanian, "A modified hybrid differential evolution for short-term scheduling of hydrothermal power systems with cascaded reservoirs," Energy Conversion and Management (2008), vol. 49, pp. 2513-2521

[9] K.K. Mandal, N. Chakraborty, "Differential evolution technique-based short-term economic generation scheduling of hydrothermal systems," Elec. Power Syst. Res. (2008), vol. 78, pp. 1972-1979.

[10] R.K. Swain, A.K. Barisal, P.K. Hota, and R. Chakrabarti, "Short-term hydrothermal scheduling using clonal selection algorithm," Electrical Power \& Energy Systems (2011), vol.33, pp.647-656.

[11] X. S. Yang, and S. Deb, "Cuckoo search via Lévy flights," In Proc. World Congress on Nature \& Biologically Inspired Computing (NaBIC 2009), India, 2009, pp.210-214.

[12] V.N. Dieu, Peter Schegner, and W. Ongsakul, "Cuckoo search algorithm for non-convex economic dispatch," IET Generation, Transmission \& Distribution (2013), vol.7, pp.645-654

[13] N.T. Thang, V.N. Dieu, T.V. Anh, "Cuckoo search algorithm for short-term hydrothermal scheduling," Applied Energy (2014), Vol. 132, pp. 276-287.

[14] N.T. Thang, V.N. Dieu, D.T. Tam, "Cuckoo Search Algorithm Using Different Distributions for ShortTerm Hydrothermal Scheduling with Cascaded Hydropower Plants," TENCON 2014- IEEE Region 10 Conference, pp. 1-6.

[15] S. Walton, O. Hassan, K. Morgan, M.R. Brown, "Modified cuckoo search: A new gradient free optimisation algorithm", Chaos, Solutions \& Fractals, (2011), vol. 44, pp. 710-718.

[16] J. Piechocki, D. Ambroziak, A. Palkowskib, G. Redlarski, "Use of Modified Cuckoo Search algorithm in the design process of integrated power systems for modern and energy self-sufficient farms," Applied Energy (2013), vol. 114, pp. 901-908.

[17] N.T. Thang, V.N. Dieu, "Modified Cuckoo search algorithm for short-term hydrothermal scheduling," Electrical Power and Energy Systems (2015), vol. 65, pp. 271-281.

[18] W.Ying, Z. Jianzhong, Z.Chao, W.Yongqiang, Q.Hui, L.Youlin. “An improved self-adaptive PSO technique for short-term hydrothermal scheduling". Expert Systems with Applications (2012), vol. 39, pp. 2288-2295.

[19] M.Basu. "An interactive fuzzy satisfying method based on evolutionary programming technique for multi-objective short-term hydrothermal scheduling”. Electric Power Systems Research (2004), vol. 69, pp. $277-285$.

[20] M. Basu. "Economic environmental dispatch of hydrothermal power system". Electrical Power and Energy Systems (2010), vol. 32, pp. 711-720.

[21] K.K. Mandal, M. Basu, N. Chakraborty. "Particle swarm optimization technique based short-term hydrothermal scheduling”. Applied Soft Computing (2008), vol. 8, pp. 1392-1399.

\section{Authors}

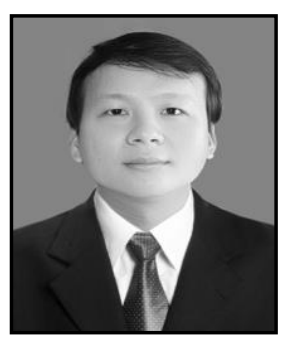

Thang Trung Nguyen, he received his B.Eng. and M.Eng degrees in Electrical Engineering from University of Technical education Ho Chi Minh City, Ho Chi Minh city, Vietnam in 2008 and 2010, respectively. Now, he is teaching at department of electrical and electronics engineering, Ton Duc Thang University, Ho Chi Minh city, Vietnam. His research interests include optimization of power system, power system operation and control and Renewable Energy.

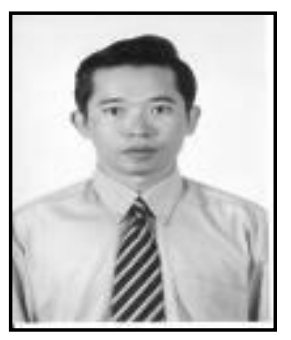

Dieu Ngoc Vo, he received B.Eng. and M.Eng. degrees in electrical engineering from HCM City University of Technology, Vietnam, in 1995 and 2000, respectively and his D.Eng. degree in energy from Asian Institute of Technology (AIT), Pathumthani, Thailand in 2007. He is now teaching at Faculty of Electrical and Electronic Engineering, HCM City University of Technology, Vietnam. His interests are applications of AI in power system optimization, power system operation and control, power system analysis, and power system. 